\title{
A client-centered relational framework on barriers to the integration of HIV and substance use services: a systematic review
}

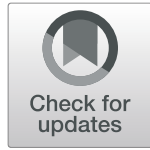

\author{
Rogério Meireles Pinto ${ }^{*}$ (D) Yun Chen and Sunggeun ( Ethan) Park
}

\begin{abstract}
Background: Given the close connection between human immunodeficiency virus (HIV) infection and substance use disorder (SUD), access to integrated HIV and SUD services is critical for individuals experiencing both challenges and their biopsychosocial conditions.

Method: Adopting an integrative method, this systematic review included 23 empirical studies published between 2000 and 2018. Articles investigated providers' and clients' perspectives on barriers to accessing integrated HIV and SUD services in various service settings (e.g., HIV primary care, SUD treatment, pharmacy).

Results: Using a client-centered relational framework, we identified barriers in three relational domains with "the client" as the focus of each: client-provider, client-organization, and client-system. The review shows that (1) barriers to HIV and SUD services do not exist in isolation, but in the dynamics within and across three relational domains; (2) service providers and clients often have different perceptions about what constitutes a barrier and the origin of such barriers; and (3) interprofessional and interorganizational collaborations are crucial for integrating HIV and SUD services.

Conclusion: This review points out the limitations of the conventional paradigm grouping barriers to service integration into isolated domains (client, provider, organization, or system). Reforms in service arrangements and provider training are recommended to address barriers to integrated services.
\end{abstract}

Keywords: HIV, Substance use, Service integration, Barriers, Client-centered relational framework

\section{Background}

Substance use disorders (SUDs) are common among people living with HIV (PLWH). People who inject drugs (PWID) or have SUDs are more likely to be exposed to and become infected by HIV and experience a more accelerated HIV progression [1-5]. Even for those individuals for whom substance use is not the transmission route for HIV, the pervasive stigma experienced by PLWH sometimes results in substance use as a coping strategy [6]. Given this close relationship between HIV and SUD, it is critical that both prevention and treatment services aiming to address the two conditions be integrated to achieve optimal health outcomes $[7,8]$. A robust collection of literature exists on the integration of HIV and SUD treatment services, which focuses on describing the advantages of

\footnotetext{
* Correspondence: ropinto@umich.edu

School of Social Work, University of Michigan-Ann Arbor, 1080 South University Avenue, Ann Arbor, MI 48109, USA
}

myriad services combinations, such as SUD counseling in HIV medical care, HIV testing in SUD outreach services, or introducing pre-exposure prophylaxis (PrEP) in SUD treatment [9-12]. Regrettably, the literature also shows that people with HIV and SUDs face myriad barriers accessing and using either or both HIV and SUD services $[13,14]$. These barriers affect and, in turn, are affected by how service consumers ("clients") interact with service providers (e.g., mutual distrust between providers and clients), with the organizations where they receive services (e.g., distant location, lack of transportation), and with the social systems (e.g., stigmatization and disenfranchisement) they navigate in order to find the HIV and SUD services they need [15-17].

Many studies draw from the service providers' or clients' data to identify barriers at the interpersonal, organizational, and system/structural levels. However, the interpretation of barriers to HIV or SUD services as isolated entities at

(c) The Author(s). 2019 Open Access This article is distributed under the terms of the Creative Commons Attribution 4.0 International License (http://creativecommons.org/licenses/by/4.0/), which permits unrestricted use, distribution, and 
different unrelated domains may obscure the fact that barriers are relational and exist in the intersection of client and provider, client and organization, and client and systems. Using a relational approach to guide our systematic review, we identified barriers in each of those intersections, which are more specific to the difficulties that clients face when trying to navigate multiple domains of services in order to address their HIV and SUD needs. Factors that hinder access to these services have been identified, and effective integrated interventions have been recommended [18-21]; however, to the best of our knowledge, little effort has been made to understand the multilayered factors that may hinder service access and utilization from a relational lens.

This integrative review includes 23 articles published between 2000 and 2018 which investigated providers' and clients' perspectives on HIV and SUD service barriers in various service settings (e.g., primary care, HIV clinics) in the U.S. As practitioners and researchers on HIV and SUD, our emphasis here is on service integration, and not one or another type of service-we aimed instead to address the important-yet-understudied issue of integration. While acknowledging the merits of a broader approach looking at specific intervention models, our review is designed to provide more specificity and depth; it thus helps to narrow an important gap in the literature, as follows. The review provides evidence supporting our assumption that barriers to HIV and SUD services do not exist in isolation; rather, these barriers are interrelated and thus inform how clients relate to their service providers and healthcare systems. This review also highlights how service providers and clients often have different perceptions about the definition and source of barriers to healthcare.
Based on our findings, we provide recommendations, specifically regarding the importance of personcentered approach and interprofessional and interorganizational collaborations, for future research and for improving best practices.

\section{Relational conceptual framework}

Both the ecological and systems theories [22, 23] suggest that a myriad of inter-connected barriers to access and delivery of biopsychosocial services exist for clients who are seeking care. Figure 1 illustrates the domains within which these barriers can be found: client, organizational (including providers), and structural/system levels. However, many barriers to access and delivery (for example, housing instability) have been conceptualized as a clientlevel issue when, in fact, housing is a structural issue affecting millions of people in the U.S. [24]. Similarly, given the popularity of the rational patient model, other structural-level (e.g., job insecurity and limited service availability) and organizational-level (e.g., long waitlist, lacking flexible service hours) barriers have been conceptualized as client-level issues (e.g., chaotic and unhealthy lifestyles, noncompliance), manifested in the common perceptions of clients' lacking ability or will to making rational decisions on their health and social care [25-27]. This fails to describe the intricate and relational nature of the barriers impeding clients from accessing health and social services. For this systematic review, we were guided by a relational approach that directs focus to the relationship between clients and their providers and service organizations, and to the socioeconomic structures under which they live.

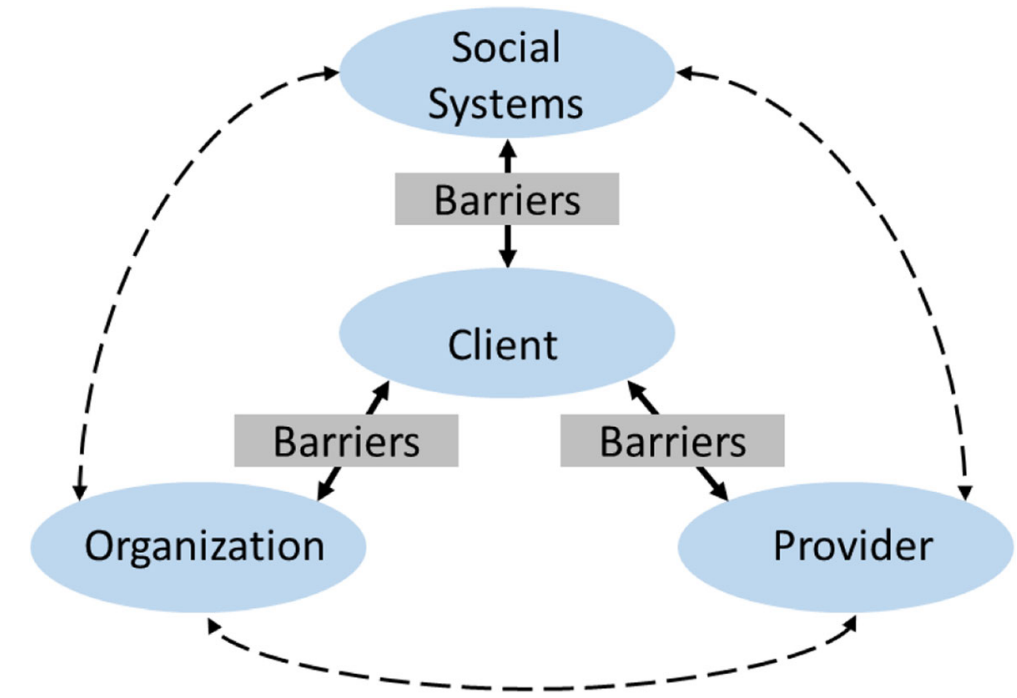

Fig. 1 Barriers to service integration — a client-centered relational framework. Guided by a client-centered relational approach, this study directs focus to the relationships between clients and their providers, clients and service organizations, and clients and the socioeconomic structures under which they live. Barriers residing in these relationships affect the dynamic and ecological interactions when clients enter a service system 
Clients have direct and indirect relationships with providers (in organizations), with organizations (within systems), and with social structures and systems. Social, economic, and political environments may introduce multiple barriers to access and delivery of HIV and SUD services. Growing evidence on how social and physical environments contribute to health inequality emphasizes the importance of understanding access/delivery from a relational lens [28]. This relational lens allows us to imagine barriers to access (e.g., poverty, lack of social support/capital), usually attributed to clients, as structural inequalities (e.g., inadequate safety nets, residential segregation, and mass incarceration minorities). Misaligned interests between clients and organizations also influence access/delivery of HIV and SUD services. For example, resource-deprived organizations may prioritize resource acquisition over services and programs addressing clients' access, such as discontinuing less profitable services or laying off specialized workforce rather than expanding service lines to address both HIV and SUD issues or hiring specialized care coordinators [29, 30].

Mismatched or contentious relationships between clients and providers often follow from front-line service provider's position to simultaneously address clients' multifaceted service needs, providers' limited capacity and resources, and incentives and rules imposed by organizations and institutions [31, 32]. For example, lack of staff training on how to work with co-morbid conditions (e.g., HIV and SUD) may affect negatively how providers work with such clients or encourage providers to allocate limited resources for addressing simpler cases that yield greater impacts-resulting in unresponsive and disrespectful care experiences for clients. Resource-deprived and unexperienced providers may make care decisions based on scientific evidence around provider-driven care, rather than engaging clients into care decisions and adjusting interventions to their circumstances and preferences-i.e., patient-centered care [31, 32]. Due to information gaps and possibly client's negative attitudes toward unresponsive care systems, a client's repeated noncompliance to a prescribed plan, perhaps justifiably due to lack of resources, may confirm providers' beliefs and stereotypes about that client-further deepening mutual distrust and hindering the client's access to life-saving health and social services (Park ES: Beyond patient-centred care: a conceptual framework of co-production mechanisms with vulnerable groups in health and social service settings, forthcoming).

\section{Methods}

This systematic review is grounded in the relational framework above and guided by an integrative review method [33] including specification of the purpose of the review, search of the extant literature and evaluation of primary sources, specification of an analytical strategy, and presentation of findings in a practical and user-friendly manner.

\section{Literature search procedures}

We conducted literature searches on EBSCOhost and Web of Science through the University of Michigan's library for the years 2000-2018. Acknowledging the fast developments in intervention science in the field of harm reduction-e.g., broad implementation of HIV prevention pre-exposure prophylaxis-we restricted our review to the past two decades, in order to exclude interventions that are outdated while capturing the most contemporary trends in service delivery. Recognizing that some articles in PubMed might not be included in either EBSCOhost or Web of Science, we also searched PubMed in a separate search using the same terms and following the same screening procedures. To improve the likelihood of finding articles about barriers to services at the intersection of the two fields, we searched for articles about HIV and SUD services separately and together, using the following permutations:

(a) Abstract (in EBSCOhost) and topic (in Web of Science): "substance abuse" OR "substance use" OR "substance misuse" OR "drug addiction" OR "drug abuse" OR "drug use" OR "drug users" OR "substance users."

(b) Abstract (in EBSCOhost) and topic (in Web of Science): "HIV prevention" OR "PrEP" OR "antiretroviral" OR "Pre-Exposure Prophylaxis" OR "HIV treatment" OR "PLWH."

(c) Abstract (in EBSCOhost) and topic (in Web of Science): "service" OR "treatment" OR "intervention" OR "care."

(d) Abstract (in EBSCOhost) and topic (in Web of Science): "access" OR "utilization" OR "adherence."

(e) Title or abstract (in EBSCOhost), and title or topic (in Web of Science): "barriers."

The search terms (a) and (b) were each combined with (c), (d), and (e) as two separate searches for barriers to HIV and SUD services, respectively, as well as together, combined with (c), (d), and (e) for barriers at the intersection of the two fields. We searched for the term "barriers" in the title for both databases in the HIV and SUD separate searches and the abstract (EBSCOhost), or as a topic (Web of Science) in the HIV/SUD combined search.

We used the online platform Rayyan QCRI (https:// rayyan.qcri.org/) [34] for managing the initial search results, including removing duplicates, removing articles that clearly fell beyond the scope of our review, and conducting the title and abstract screening of all articles that fulfilled our initial inclusion criteria. This online platform allowed two of our collaborators to blind review all the titles and abstracts independently. 


\section{Inclusion and exclusion criteria}

In the initial screening, we included peer-reviewed studies on the barriers to service access in the intersection of HIV prevention and care and SUD services in the United States (U.S.). We excluded papers that were (1) not about the U.S., (2) not about the intersection of the two fields, (3) reviews or commentary articles, and (4) clinical trials or evaluations of particular service programs. After this initial round of titles and abstract screening, the three authors held multiple face-to-face discussions and agreed to exclude studies that were (5) only on the adherence to treatment or medication of one specific substance. Since our review focuses on service practices, we also excluded studies that (6) analyze administrative or policy documents. In order to be readily able to use the review's findings to guide practice and policy development, we focused the search on the U.S. because different countries have different healthcare and social service systems and thus myriad different barriers.

\section{Article selection procedures}

Figure 2 summarizes the article selection procedures. Our initial search of EBSCOhost and Web of Science yielded 483 U.S.-based articles. Using our inclusion/exclusion criteria, on our first-round title and abstract screening, we excluded 413 papers. Then, after the initial screening, we agreed to exclude studies that exclusively focused on adherence since it entails questions characteristically different from those of service access and utilization. This left us with 27 articles to consider for further review. We did an initial categorization of the 27 articles based on title, abstract, and method, and screened out three (two policy/administrative analysis and one without specific service setting information) (n $=24$ ). Following the same searching and screening procedure, the additional search on PubMed yielded two qualifying articles, resulting in a total of 26 articles for initial full-text coding. After the initial coding of these 26 articles, we further identified three papers that we agreed to exclude because they turned out to be outside the scope of the review. Two of the three articles did not center on barriers to service access and utilization [35, 36 , and one article solely focused on client's individuallevel barriers (e.g., denial of HIV diagnosis, forgetting medication, or prioritizing drug use over HIV treatment) [5]. The final selection included 23 articles that empirically examined the barriers to HIV and SUD service access from the perspectives of providers and clients in myriad service settings in the U.S.

\section{Article analysis}

Guided by an integrative review method [33], we used a published data analysis plan developed by the Pinto and colleagues [37], which included purposive sampling, a modified version of grounded theory, and collaborative interpretation [38]. "Purposive sampling" here is used to describe the above procedures we used to select the articles for this review-specific search terms and inclusion and exclusion selection criteria. Our analysis reflects a modified version of the grounded theory [39] in that we selected the final set of articles grounded in our experiences as researchers and practitioners who have provided both HIV and SUD services. We used a collaborative approach, whereas each chosen article was examined independently by at least two of the authors and discussed by all three. Our lengthy (average $2 \mathrm{~h}$ ) and frequent (average weekly) discussions were used to address researcher bias and to reach $100 \%$ agreement on selected papers and the final results (reflected in Table 1). Our analysis consisted of the following steps:

- Step 1-We extracted all information related to service barriers from each article.

- Step 2a-We categorized barriers at the interpersonal, organizational, and system levels.

- Step 2b-We examined each barrier selected from step $2 \mathrm{a}$ and held discussions on how to reconceptualize them grounded in our relational framework.

- Step 3-We categorized all barriers to reflect a client-centered relational framework highlighting the dynamic relationships between clients and service providers, organizations, and social systems.

\section{Results}

Table 1 shows our final set of 23 articles, which includes empirical studies on barriers to integration of HIV and SUD services, including data from service providers $(n=$ $10)$, clients $(n=11)$, and both clients and providers $(n=$ 2). Thirteen of the 23 articles used quantitative methods or secondary survey data analysis, nine used qualitative interviews or case studies, and one used a mixedmethods approach. Twelve studies were conducted in HIV primary care settings, four in SUD treatment settings, five drew samples from multiple settings including healthcare clinics and community-based organizations, and two from "other settings."

The reviewed articles highlight service integration aimed to help individuals facing myriad challenges related to both HIV exposure and SUD. Several studies recommended the integration of services in HIV primary care settings for clients who also inject drugs. Proposed modalities of service integration included having primary care providers distribute sterile syringes [48], providing methadone and buprenorphine treatments [44, 47], strengthening clients' social supports [57], and addressing sociodemographic obstacles to care and competing health needs [42]. Regarding service integration in SUD treatment settings, this review highlights 
Potentially relevant studies based on keyword search in Web of Science \&

EBSCOhost $(\mathrm{n}=1342$, with duplicates)

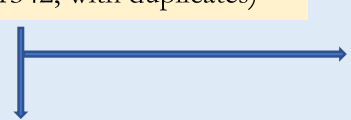

Remove duplicates and non-US

studies $(n=859)$

US-based studies $(\mathrm{n}=483)$

Excluded articles:

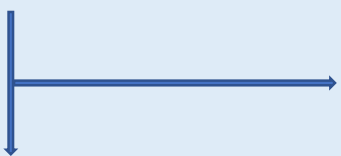

1) Not on service barriers

2) Not on the intersection of the

HIV \& SUD

3) Reviews and commentaries

4) Clinical trials or program

evaluations

US-based studies on the barriers to HIV

$(\mathrm{n}=413)$ and SUD services

$(\mathrm{n}=70)$

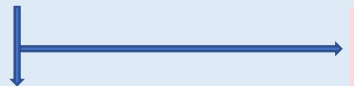

Excluded articles:

1) Treatment or medication

US-based studies on the barriers to HIV adherence $(n=43)$

and SUD services access and utilization $(\mathrm{n}=27)$

Excluded articles:

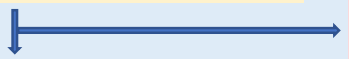

1) Policy or administrative document analysis

US-based studies on the barriers to HIV \& SUD service access and utilization:

2) Not specifying service settings $(\mathrm{n}=3)$

providers' or clients' perspectives, specified service settings $(n=24)$

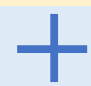

PubMed search: 2 more articles met our inclusion criteria $(\mathrm{n}=2)$

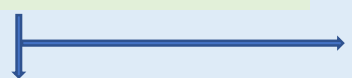

3 articles further excluded after firstround of full-text analysis given that they diverged from the scope of this

Final Sample: US-based studies on the review $(\mathrm{n}=3)$

barriers to HIV \& SUD service access and utilization from providers' or clients' perspectives in specified service settings $(n=23)$

Fig. 2 Summary of article selection and inclusion/exclusion criteria. Based on a comprehensive search and screening of literature in Web of Science, EBSCOhost, and PubMed, the study identified 23 articles that empirically examined the barriers to HIV and SUD service access from the perspectives of providers and clients in myriad service settings in the U.S.

the need for medical treatment access for HIV-positive injection drug users receiving services in community-based settings [56, 57]. The review also highlights the need for integrating preventive services such as HIV testing [58] and pre-exposure prophylaxis (PrEP) $[10,53,54,59]$ into community-based facilities. This would improve service availability, accessibility, and connectivity for SUD clients who are exposed to environments where HIV is prevalent.

Table 1 reveals common themes that emerged from selected articles regarding barriers to service within three domains of reference. Below, we present a summary of barriers in each domain to highlight unique client-centered dynamic relationships with service providers, organizations, and social systems, reflecting the conceptual frame provided above.

\section{Client-provider relational domain}

Barriers under this domain were grouped as (a) provider concerns or biases toward clients, (b) provider lacking competencies, and (c) poor provider-client relationship. 


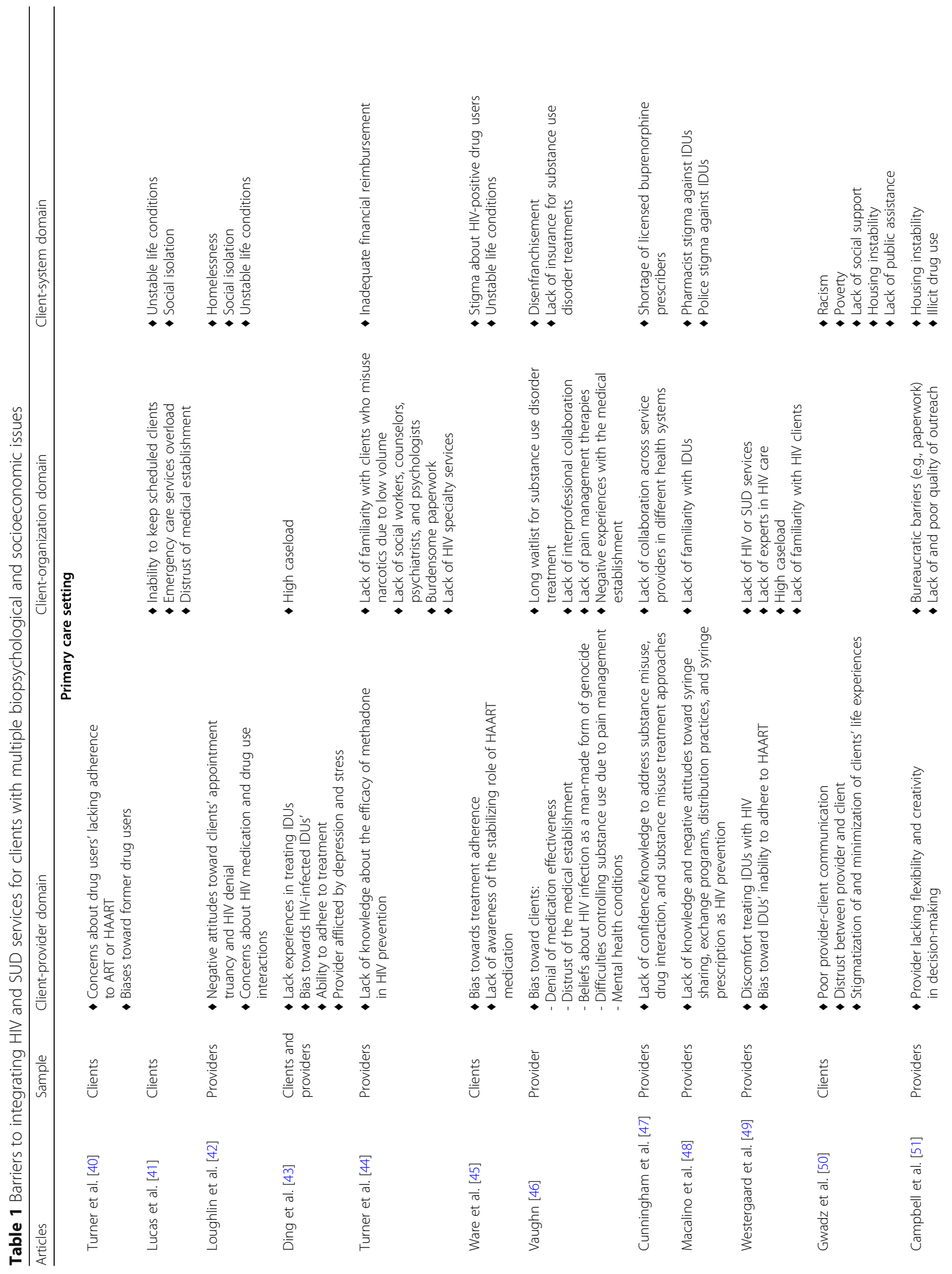




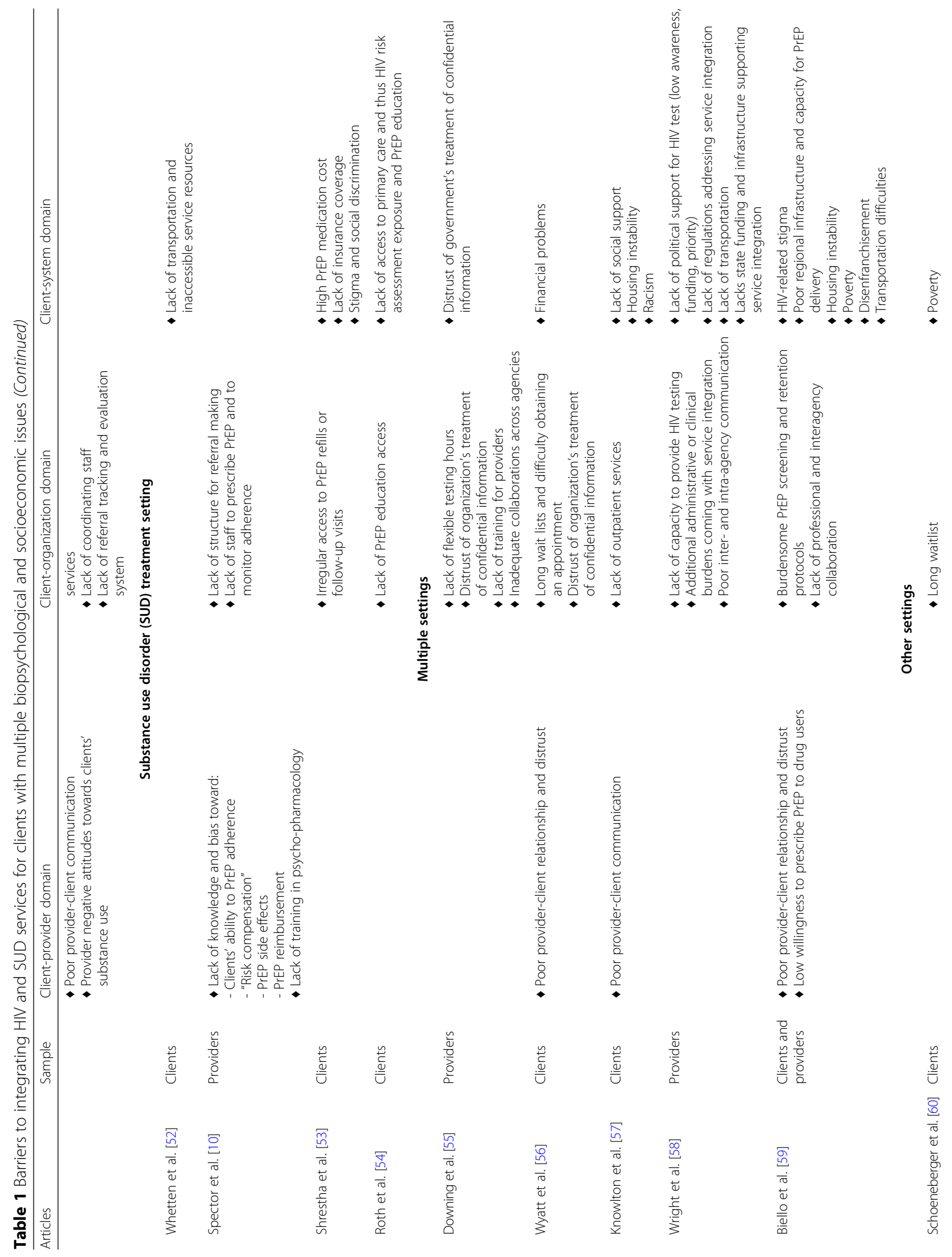




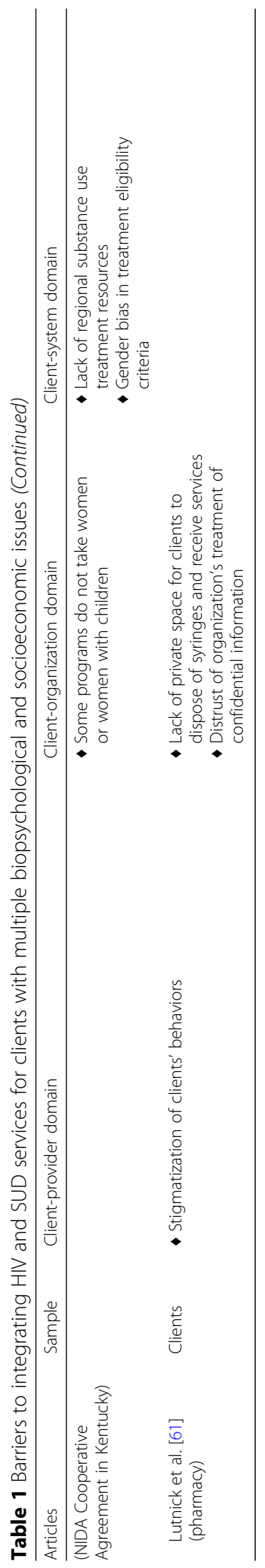


Many articles investigating the client's perspective identified providers' biases and concerns about clients' characteristics-for example, clients' difficulties in adhering to medical treatments, lack of readiness to initiate treatment, denial of HIV status, or inability to keep appointments [40, 42, 43, 45, 46, 49-51, 61]. Another type of client-provider barrier was providers' lack of awareness, knowledge, skills, experience, or confidence in serving clients diagnosed with co-morbidities and/or in carrying out specific treatment models [10, 43, 44, 47, 48, 51]. Poor provider-client relationships, including poor communication, confidentiality issues, and mutual distrust, were identified in many articles [46, 50, 51, 56, 57, 59]. One study also pointed out providers' mental health status and stressful life conditions as a potential barrier [43], and thus highlighted the need of self-care among providers for carrying out services effectively.

\section{Client-organization relational domain}

Barriers under this domain were grouped based on organizational issues: (a) inconvenient procedures for clients; (b) challenges for providers, such as lack of service experience and competency; and (c) lack of interprofessional and/or interagency collaborations. These clinical and administrative issues may cause difficulties for both clients to access and for providers to deliver services. Examples include inflexible HIV testing hours [55], long waitlists $[46,56,60]$, difficulty in obtaining appointments [56], and lack of regular medication refills or follow-up visit arrangements [53]. Our review identified the following key barriers within the client-organizational domain: lack of training resources or low training quality $[54,55]$, high caseload [43, 49], and burdensome paperwork [44, $51,59]$. Other key barriers include lack of access to consultants and services from other professions [10, 44, 46, $47,59]$, ineffective interagency referral structure $[10,51]$, and poor inter- and intra-agency communication [51, 58]. This review found that some HIV clinics were in areas with a relatively lower volume of SUD clients; thus, providers in these clinics had limited knowledge and experiences of working with dual-diagnosis clients [44, 48, 49].

\section{Client-system relational domain}

Barriers under this domain were grouped based on structural issues that lead to (a) socioeconomic and health-related disparities for clients, (b) deficiencies in resources and social support available to clients, and (c) stigmatization and discrimination of clients' behaviors and socioeconomic conditions. The review found that individuals with both HIV and SUDs may face poverty, homelessness, social instability, and an unfair criminal justice system [41, 42, 50, 51, 59, 60]. Collectively, these conditions contribute to further barriers, such as multiplication of competing demands $[10,45,56]$, and are particularly related to late access of HIV and/or SUD treatment [49]. Deficiencies in resources and supports include insurance coverage, transportation, public assistance, treatment options, and social support $[46,50,52-$ 54, 56-60]. Unequal treatment and inequitable resource distribution $[44,47,58]$, such as insufficient financial reimbursements [44], directly affect individuals diagnosed with co-morbidities like HIV and substance use disorders. Moreover, individuals with both HIV and SUD also face social, economic, and political stigmatization and discrimination in the form of racism, gender biases, and stereotypes $[45,48,50,53,57,59,60]$. Structural stigmatization and discrimination may lead to negative experiences in service systems $[46,59]$ and, even worse, may block initial entry to service systems $[42,50,60]$.

\section{Discussion}

This review of 23 empirical studies identified a myriad of barriers to accessing integrated HIV and SUD services in different healthcare settings. We grounded our analysis of selected papers in a relational framework suggesting that barriers can be best understood and therefore addressed by linking them to the relationships that exist between clients and providers, clients and organizations, and clients and systems. This perspective shifts the origin of access barriers to where they belong. For example, housing instability is an access barrier often discussed as a client's problem (e.g., lack of work ethic). Alternatively, homelessness can be understood as a conflict between clients and socio-economic and political structures. As many clients with HIV and SUD issues face difficulties in finding stable jobs and income sources, they are more likely to experience housing instability, which in turn creates further barriers to employment to access to HIV and SUD treatments. From a relational perspective, such service barriers can be better understood as conflicts between client and structures requiring macro-level policy and program interventions, rather than issues pertaining exclusively to the clients (e.g., lack of will or capacity) or providers (e.g., lack of respect for clients).

We note that 21 out of the 23 reviewed articles reported barriers in more than one domain and eight articles reported barriers in all three domains. This finding illustrates that barriers to the access and delivery of integrated HIV and SUD services should not be treated as isolated issues pertaining solely to clients, providers, organizations, or systems. Rather, barriers in one domain often co-exist with barriers in the other domains. For example, in the case study by Vaughn [46], the unequal distribution of resources (e.g., certain populations were disenfranchised from basic social and medical establishments) and its negative impacts on the clinical and administrative operations of service organizations (e.g., long waitlist, limited eligibility criteria, and the lack of 
interprofessional services) directly contributed to the disadvantaged conditions experienced by the clients and to the negative relationships between clients and service providers. Another example from Campbell and colleagues [51] study showed that the unstable life conditions and complex challenges faced by clients with dual diagnoses required providers to be more aware of and knowledgeable about the need for and the mechanisms of integrating HIV and SUD treatment services. However, providers' awareness and ability to integrate services may be hindered by organizational factors, such as the lack of coordinating staff, interagency collaboration, and burdensome administrative tasks. The relational approach highlights the need for treating barriers, identified at one level or another, as dynamic and interconnected. Therefore, we recommend that interventions addressing such barriers involve different actors (e.g., clients and providers) address two or more domains. The fact that we observed similar sets of barriers across service settings in both decades of research suggests that very little change has occurred in the client's relationships with provider, organization, and system in the last two decades. This suggests the need for more systemic interventions focusing on the intersection of diverse stakeholders.

Our relational framework also challenges narratives attributing client's limited access and adherence to integrated HIV and SUD services as being client-level issues. Research shows that injection drug users and other SUD clients can successfully undergo HIV treatment [62]. Notwithstanding, our review suggests that structural inequalities (e.g., lack of transportation and social resources) are at the root of clients' unstable and often chaotic lives. These structural problems thus put clients at a disadvantage and render them unable to access services. Krüsi and colleagues [16] point out the overestimation of individual/ behavioral-level variables and underestimation of social and structural factors in the extant literature. This perception appears also to influence providers to perceive their clients as lacking the capacity to acknowledge their problems, initiate services, or adhere to treatments [10, 42, 46]. However, here we do not intend to blame providers or to imply that all providers are ignorant of the organizationalor structural-level barriers. We acknowledge the complexities in practice that providers often are limited in what they can do to help with structural barriers such as stigma and racism. Calling out the discrepancy in causal attributions is to highlight clients' perspectives and their lived experiences as important considerations in service designs and implementations. Interventions aiming to address these issues will need to focus on structural factors in order to develop a base upon which clients can begin to address their individual-level problems.

Fragmented and competitive funding streams and limited political and technical supports can discourage organizations from addressing clients' dual HIV and SUD issues comprehensively [29]. Integration of HIV and SUD services may be accomplished by facilitating providers' collaborations within and beyond organizational boundaries. Regrettably, very few articles in this review provided replicable models or systems of service integration. Our review does highlight the need for greater interprofessional and interorganizational collaborations for promoting clients' access to both HIV and SUD treatment services [62]. Some articles [44, 47, 59] reported the lack of collaboration and knowledge sharing among service providers. Therefore, we recommend that "collaboration"-the flow of knowledge and expertise across professional and organizational boundaries-be spelled out through institutional arrangements and structural supports. These would be reinforceable behaviors with the potential to facilitate effective communications, sharing responsibilities, and integration of services [7, 8, 62].

This review found that some HIV clinics are located in areas with a relatively lower volume of SUD clients. Providers in these settings, less exposed to clients with SUD, had limited knowledge and experiences that they can use to inform their work with dual-diagnosis clients. This lack of exposure and knowledge may be a contributor to providers' biases and stereotypes about clients. Similarly, providers lacking expertise and training to mitigate this gap might experience more stress and concerns about their skillset that may exacerbate relational problems with their clients. We have not found empirical evidence among the articles in the review to confirm this position; however, it is worth noting one study that suggested providers' mental health status and stressful life conditions as a potential barrier to access and delivery of services [43]. This highlights the need for further research in this area and intervention development to improve self-care among providers. Social support attained through interprofessional collaboration training is highly recommended.

\section{Conclusion}

In this paper, we proposed and applied a client-centered relational framework in order to identify barriers to the integration of HIV and SUD services. This approach highlights the dynamic interactions between clients and providers, organizations, and systems from which they can access the services they need. Instead of examining barriers on one or another domain, we examined challenges that clients with HIV and SUDs need to face visà-vis service providers, organizations, and structures. This process highlighted not only the central role of clients but also the interconnectedness of barriers across different domains. The relational framework also helps service organizations and providers to identify areas for improvement. When zooming in on barriers in particular levels, it is easy to blame clients' individual attributes 
and behaviors, but doing so may result in stigmatizing and unjust practices. By taking a client-centered view, the relational framework helps providers and organizational leaders to recognize gaps in services and identify ways to address barriers to integration and not in isolation. This integrated approach might include training providers and managers in interprofessional and interorganizational collaboration.

\section{Acknowledgements}

None.

\section{Authors' contributions}

The research question and overall research design were collaboratively generated and defined by all three authors. YC and SEP conducted coding. Under RMP's supervision, the authors met regularly, discussed, and revised the codes throughout several rounds of the analysis. All three authors drafted substantial portions of the manuscript and worked on the revisions. All authors read and approved the final manuscript.

\section{Funding}

Nothing declared.

\section{Availability of data and materials}

The data supporting the conclusions of this article are all published academic journal articles. They are available in Web of Science, EBSCOhost, and PubMed.

\section{Ethics approval and consent to participate}

Not applicable. This is a systematic review utilizing published academic journal articles as our data for analysis.

\section{Consent for publication}

Not applicable.

\section{Competing interests}

The authors declare that they have no competing interests.

Received: 7 December 2019 Accepted: 10 December 2019

\section{Published online: 19 December 2019}

\section{References}

1. Centers for Disease Control and P. HIV Surveillance Report 2017. Atlanta, GA: CDC; 2018 2018/11//. Report No.: vol. 29.

2. Dash S, Balasubramaniam M, Villalta F, Dash C, Pandhare J. Impact of cocaine abuse on HIV pathogenesis. Front Microbiol. 2015;6.

3. Galvan FH, Burnam MA, Bing EG. Co-occurring psychiatric symptoms and drug dependence or heavy drinking among HIV-positive people. J Psychoactive Drugs. 2003;35(Suppl 1):153-60.

4. Hauser KF, Knapp PE. Interactions of HIV and drugs of abuse: the importance of glia, neural progenitors, and host genetic factors. International Review of Neurobiology. 2014;118:231-313.

5. Kuchinad KE, Hutton HE, Monroe AK, Anderson G, Moore RD, Chander G. A qualitative study of barriers to and facilitators of optimal engagement in care among PLWH and substance use/misuse. BMC Res Notes. 2016;9(1). https://doi.org/10.1186/s13104-016-2032-4.

6. Levi-Minzi MA, Surratt HL. HIV stigma among substance abusing people living with HIV/AIDS: implications for HIV treatment. AIDS Patient Care STDs. 2014;28(8):442-51.

7. Altice FL, Kamarulzaman A, Soriano W, Schechter M, Friedland GH. Treatment of medical, psychiatric, and substance-use comorbidities in people infected with HIV who use drugs. Lancet (London, England). 2010; 376(9738):367-87.

8. Zaller N, Gillani FS, Rich JD. A model of integrated primary care for HIVpositive patients with underlying substance use and mental illness. AIDS care. 2007:19(9):1128-33.

9. Pollack HA, D'Aunno T. HIV testing and counseling in the nation's outpatient substance abuse treatment system, 1995-2005. J Subst Abuse Treat. 2010;38(4):307-16.
10. Spector AY, Remien RH, Tross S. PrEP in substance abuse treatment: a qualitative study of treatment provider perspectives. Subst Abuse Treat Prev Policy. 2015;10(1). https://doi.org/10.1186/1747-597X-10-1.

11. Springer SA, Chen S, Altice FL. Improved HIV and substance abuse treatment outcomes for released HIV-infected prisoners: the impact of buprenorphine treatment. J Urban Health. 2010;87(4):592-602.

12. Brown LS, Kritz SA, Goldsmith RJ, Bini EJ, Rotrosen J, Baker S, et al. Characteristics of substance abuse treatment programs providing services for HIV/AIDS, hepatitis C virus infection, and sexually transmitted infections: The National Drug Abuse Treatment Clinical Trials Network. J Subst Abuse Treat. 2006;30(4):315-21.

13. Burnam MA, Bing EG, Morton SC, Sherbourne C, Fleishman JA, London AS, et al. Use of mental health and substance abuse treatment services among adults with HIV in the United States. Arch General Psychiatry. 2001;58(8): 729-36.

14. Mathers BM, Degenhardt L, Ali H, Wiessing L, Hickman M, Mattick RP, et al. HIV prevention, treatment, and care services for people who inject drugs: a systematic review of global, regional, and national coverage. Lancet (London, England). 2010;375(9719):1014-28.

15. Bruce RD, Kresina TF, McCance-Katz EF. Medication-assisted treatment and HIV/AIDS: aspects in treating HIV-infected drug users. AIDS. 2010;24(3):33140.

16. Krüsi A, Wood E, Montaner J, Kerr T. Social and structural determinants of HAART access and adherence among injection drug users. Int I Drug Policy. 2010;21(1):4-9.

17. Ti L, Kerr T. Task shifting redefined: removing social and structural barriers to improve delivery of HIV services for people who inject drugs. Harm Reduction J. 2013:10(1):20

18. Altice FL, Bruce RD, Lucas GM, Lum PJ, Korthuis PT, Flanigan TP, et al. HIV treatment outcomes among HIV-infected, opioid-dependent patients receiving buprenorphine/naloxone treatment within HIV clinical care settings: results from a multisite study. J Acquir Immune Defic Syndr (1999). 2011;56(Suppl 1):S22-32.

19. Berg KM, Litwin A, Li X, Heo M, Arnsten JH. Directly observed antiretroviral therapy improves adherence and viral load in drug users attending methadone maintenance clinics: a randomized controlled trial. Drug and Alcohol Dependence. 2011;113(2-3):192-9.

20. Schroeder JR, Epstein DH, Umbricht A, Preston KL. Changes in HIV risk behaviors among patients receiving combined pharmacological and behavioral interventions for heroin and cocaine dependence. Addictive Behaviors. 2006:31(5):868-79.

21. Uhlmann S, Milloy MJ, Kerr T, Zhang R, Guillemi S, Marsh D, et al. Methadone maintenance therapy promotes initiation of antiretroviral therapy among injection drug users. Addiction. 2010;105(5):907-13.

22. Richardson GP. Feedback thought in social science and systems theory. Philadelphia, PA, USA: University of Pennsylvania; 19911991.

23. Siporin M. Ecological systems theory in social work. J Sociol Social Welfare. 1980;7:507-32.

24. Aidala A, Cross JE, Stall R, Harre D, Sumartojo E. Housing status and HIV risk behaviors: implications for prevention and policy. AIDS and behavior. 2005; 9(3):251-65.

25. Corrigan PW, Rüsch N, Ben-Zeev D, Sher T. The rational patient and beyond: implications for treatment adherence in people with psychiatric disabilities. Rehabil Psychol. 2014;59(1):85-98.

26. Cobb M, de Chabert JT. HIV/AIDS and care provider attributions: who's to blame? AIDS Care. 2002;14(4):545-8.

27. Fineman $\mathrm{N}$. The social construction of noncompliance: a study of health care and social service providers in everyday practice. Sociol Health Illness. 1991;13(3):354-74.

28. Wilkinson R, Marmot M. The solid facts: social determinants of health. 2nd ed ed. Copenhagen, Denmark: Centre for Urban Health, World Health Organization; 2003; 2003. p. 31

29. Park SE, Mosley J. Nonprofit growth and decline during economic uncertainty. Human Service Organizations: Management, Leadership \& Governance. 2017;41(5):515-31.

30. Smith SR, Lipsky M. Nonprofits for hire: the welfare state in the age of contracting. Cambridge, MA: Harvard University Press; 19951995.

31. Brodkin EZ. Reflections on Street-Level Bureaucracy: Past, Present, and Future. Public Administration Rev. 2012;72(6):940-9.

32. Lipsky M. Street level bureaucracy: dilemmas of the individual in public services: Russell Sage Foundation; 1980; 1980. 
33. Whittemore $R$, Knafl $K$. The integrative review: updated methodology. Journal of Advanced Nursing. 2005;52(5):546-53.

34. Ouzzani M, Hammady H, Fedorowicz Z, Elmagarmid A. Rayyan-a web and mobile app for systematic reviews. Systematic Reviews. 2016;5(1):210.

35. Sambamoorthi U, Warner LA, Crystal S, Walkup J. Drug abuse, methadone treatment, and health services use among injection drug users with AIDS. Drug Alcohol Depend. 2000;60(1):77-89.

36. Magidson JF, Seitz-Brown CJ, Listhaus A, Lindberg B, Anderson KE, Daughters SB. Distress tolerance and use of antiretroviral therapy among HIV-infected individuals in substance abuse treatment. AIDS Patient Care STDS. 2013;27(9):518-23.

37. Pinto RM, Berringer KR, Melendez R, Mmeje O. Improving PrEP implementation through multilevel interventions: a synthesis of the literature. AIDS and Behavior. 2018:22(11):3681-91.

38. Cypress BS. Rigor or reliability and validity in qualitative research: rerspectives, strategies, reconceptualization, and recommendations. Dimens Crit Care Nurs. 2017:36(4):253-63.

39. Strauss A, Corbin JM. Basics of qualitative research: grounded theory procedures and techniques. Thousand Oaks, CA, US: Sage Publications, Inc; 1990 1990. 270 p.

40. Turner BJ, Fleishman JA, Wenger N, London AS, Burnam MA, Shapiro MF, et al. Effects of drug abuse and mental disorders on use and type of antiretroviral therapy in HIV-infected persons. J Gen Intern Med. 2001;16(9):625-33.

41. Lucas GM, Gebo KA, Chaisson RE, Moore RD. Longitudinal assessment of the effects of drug and alcohol abuse on HIV-1 treatment outcomes in an urban clinic. AIDS. 2002;16(5):767-74.

42. Loughlin A, Metsch L, Gardner L, Anderson-Mahoney P, Barrigan M, Strathdee $S$. Provider barriers to prescribing HAART to medically-eligible HIV-infected drug users. Aids Care. 2004;16(4):485-500.

43. Ding L, Landon BE, Wilson IB, Wong MD, Shapiro MF, Cleary PD. Predictors and consequences of negative physician attitudes toward HIV-infected injection drug users. Archives of Internal Medicine. 2005;165(6):618.

44. Turner BJ, Laine C, Y-t L, Lynch K. Barriers and facilitators to primary care or human immunodeficiency virus clinics providing methadone or buprenorphine for the management of opioid dependence. Arch Internal Med. 2005;165(15):1769.

45. Ware NC, Wyatt MA, Tugenberg T. Adherence, stereotyping and unequal HIV treatment for active users of illegal drugs. Soc Sci Med. 2005:61(3):565-76.

46. Vaughn A. Substance abuse, medications, HIV, and the community. Nurs Clin North America. 2006:41(3):355-69.

47. Cunningham CO, Kunins HV, Roose RJ, Elam RT, Sohler NL. Barriers to obtaining waivers to prescribe buprenorphine for opioid addiction treatment among HIV physicians. Journal of General Internal Medicine. 2007; 22(9):1325-9.

48. Macalino GE, Sachdev DD, Rich JD, Becker C, Tan LJ, Beletsky L, et al. A national physician survey on prescribing syringes as an HIV prevention measure. Subst Abuse Treat Prev Policy. 2009:4:9.

49. Westergaard RP, Ambrose BK, Mehta SH, Kirk GD. Provider and clinic-level correlates of deferring antiretroviral therapy for people who inject drugs: a survey of North American HIV providers. Journal of the International AIDS Society. 2012;15(1):10.

50. Gwadz M, de Guzman R, Freeman R, Kutnick A, Silverman E, Leonard NR, et al. Exploring how substance use impedes engagement along the HIV care continuum: a qualitative study. Front Public Health. 2016;4.

51. Campbell ANC, Wolff M, Weaver L, Jarlais DD, Tross S. "It's never just about the HIV:" HIV primary care providers' perception of substance use in the era of "universal" antiretroviral medication treatment. AIDS and behavior. 2018; 22(3):1006-17.

52. Whetten R, Whetten K, Pence BW, Reif S, Conover C, Bouis S. Does distance affect utilization of substance abuse and mental health services in the presence of transportation services? AIDS Care. 2006;18(sup1):27-34.

53. Shrestha R, Copenhaver M. Exploring the use of pre-exposure prophylaxis (PrEP) for HIV prevention among high-risk people who use drugs in treatment. Front Public Health. 2018;6.

54. Roth AM, Aumaier BL, Felsher MA, Welles SL, Martinez-Donate AP, Chavis M, et al. An exploration of factors impacting preexposure prophylaxis eligibility and access among syringe exchange users. Sexually Transmitted Diseases. 2018:45(4):217-21.

55. Downing M, Mulia N, Vernon K, Knight K, Ferreboeuf M, Carroll A. Voices from the field: providers discuss HIV counseling and testing programs for drug users. Aids \& Public Policy Journal. 2000;15(2):48-64.
56. Wyatt GE, Carmona JV, Loeb TB, Williams JK. HIV-positive black women with histories of childhood sexual abuse: patterns of substance use and barriers to health care. J Health Care Poor Underserved. 2005;16(4):9-23.

57. Knowlton A, Arnsten J, Eldred L, Wilkinson J, Gourevitch M, Shade S, et al. Individual, interpersonal, and structural correlates of effective HAART use among urban active injection drug users. JAIDS J Acquir Immune Defic Syndr. 2006;41(4):486-92.

58. Wright $\mathrm{PB}$, Curran $\mathrm{GM}$, Stewart $\mathrm{KE}$, Booth $\mathrm{BM}$. A qualitative analysis of provider barriers and solutions to HIV testing for substance users in a small, largely rural southern state: provider barriers to HIV testing. J Rural Health. 2013: 29(4):420-431n/a-n/a.

59. Biello KB, Bazzi AR, Mimiaga MJ, Biancarelli DL, Edeza A, Salhaney P, et al. Perspectives on HIV pre-exposure prophylaxis (PrEP) utilization and related intervention needs among people who inject drugs. Harm Reduction J. 2018;15(1):55.

60. Schoeneberger ML, Logan TK, Leukefeld CG. Age differences in HIV risk behaviors and drug treatment utilization among drug users in Kentucky. Substance Use Misuse. 2001;36(6-7):867-86.

61. Lutnick A, Case P, Kral AH. Injection drug users' perspectives on placing HIV prevention and other clinical services in pharmacy settings. J Urban Health. 2012;89(2):354-64

62. Volkow ND, Montaner J. The urgency of providing comprehensive and integrated treatment for substance abusers with HIV. Health Affairs. 2011; 30(8):1411-9.

\section{Publisher's Note}

Springer Nature remains neutral with regard to jurisdictional claims in published maps and institutional affiliations.
Ready to submit your research? Choose BMC and benefit from:

- fast, convenient online submission

- thorough peer review by experienced researchers in your field

- rapid publication on acceptance

- support for research data, including large and complex data types

- gold Open Access which fosters wider collaboration and increased citations

- maximum visibility for your research: over $100 \mathrm{M}$ website views per year

At BMC, research is always in progress.

Learn more biomedcentral.com/submissions 